\title{
Evaluative Attitudes Towards Sexual Offending Against Children: The Development of Self Report Measure Items
}

\author{
By \\ Emily Dunn \\ A thesis submitted to \\ the Faculty of Graduate and Postdoctoral Affairs \\ in partial fulfillment of the requirements for the degree of \\ Master of Arts \\ in \\ Psychology \\ Carleton University \\ Ottawa, Ontario \\ (C) 2021 \\ Emily Dunn
}




\begin{abstract}
The purpose of this study is to develop a measure of evaluative attitudes towards sexual offending against children. The secondary purpose is to explore the relevance of the items I created. I created 60 items that depict sexual offending towards children. Items were administered to inmates from the Missouri Sex Offender Program in Jefferson City, MO. Participants rated items using either one of two different Likert scales of 1-4. The items were refined according to highest variance, creating the Evaluative Attitudes Towards Sexual Offending Against Children scale (EASOC). Mean scores of each offence group were compared to see if those with a current hands-on sexual offences have less negative evaluative attitudes about sexual offending. The study will explore if there is a difference in EASOC responses between men with sexual offences against children and men with other offences, and possible connection between evaluative attitudes and sexual offending against children.
\end{abstract}


Table of Contents

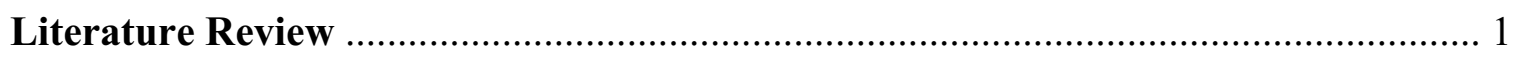

Scales Potentially Assessing Evaluative Attitudes................................4

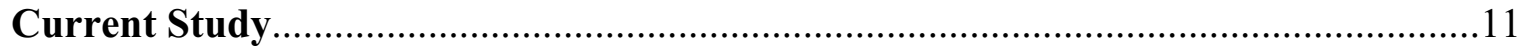

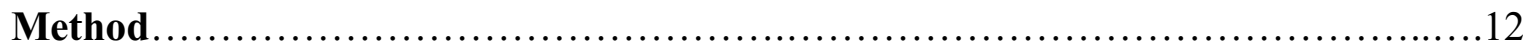

Participants....................................................... 12

Item Development.................................................. 14

Additional Measures.................................................... 16

Procedure ...................................................................

Statistical Analysis................................................... 16

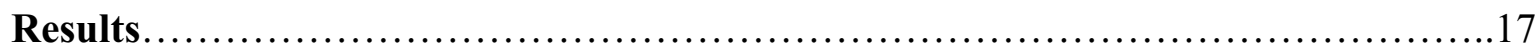

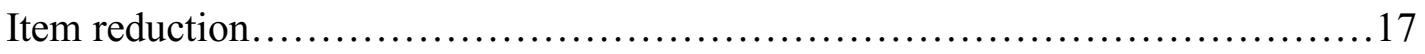

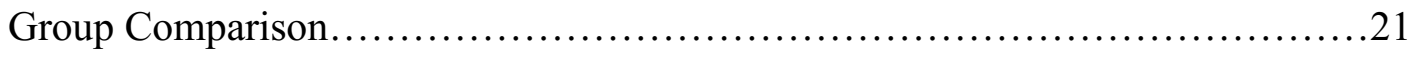

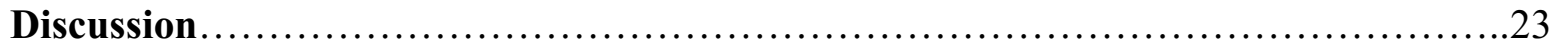

Components of The Reduced Items.................................... 25

SOC vs Non SOC: Comparing Mean Score Responses........................26

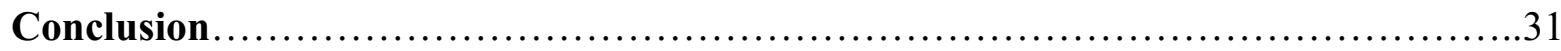

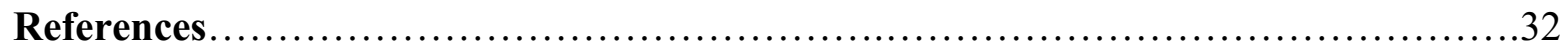

Appendix A..................................................................... 


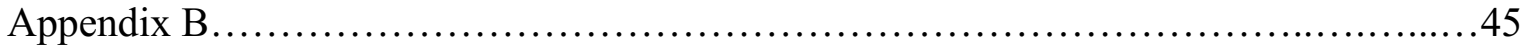

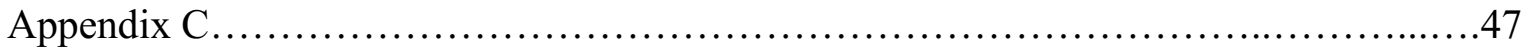

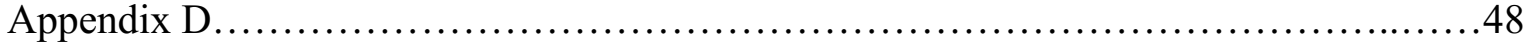




\section{List of Tables}

Table 1........................................................................................ 13

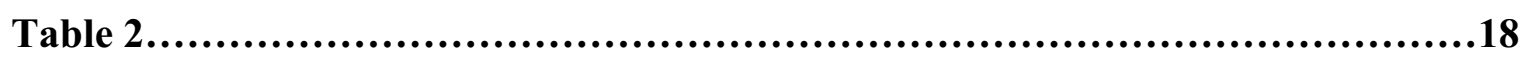

Table 3........................................................................................19 


\section{List of Appendices}

Appendix A...............................................................................36

Appendix B.............................................................................46

Appendix C..................................................................................47

Appendix D..............................................................................48 


\section{Evaluative Attitudes Towards Sexual Offending Against Children: The Development of Self Report Measure Items}

Sexual offending against children is a prominent social issue with reporting rates increasing over the years. According to the 2012 report on police-reported sexual offending against children and youth in Canada, children are more likely than adults to be victims of sexual assault, but adult sexual assault has a higher rate of police reports. From 2010 to 2017 there has been a $118 \%$ increase in police reports of sexual offences against children (Department of Justice Canada, 2017). With increasing awareness of sexual assault against children, there is increased need for research on sexual offenders for both prediction and treatment.

Sexual offenders against children are typically studied and addressed by two main domains: the academic setting, which largely consists of research and theory, and the correctional setting, which focuses on applying practical methods of prevention and treatment. Despite their differences, both domains have an interest in the two widely used constructs of cognitive distortions, and attitudes. Cognitive distortions tend to be defined as a manner of thinking that is both exaggerated and irrational (Beck, 1979). Cognitive distortions are relevant in the correctional world because many self-report measures used in these facilities reflect cognitive distortions in sexual offenders, such as the Cognitions Scale and the MOLEST Scale (Abel et al., 1989; Bumby 1996).

\section{Literature Review}

Regarding the construct of attitudes, there are several accepted definitions, the most widely used and more casual definition being "a mental position with regard to fact or state", also "a feeling of emotion towards a fact or state" (Merriam Webster, 2020, def. 4a, 4b). This definition illustrates typically how correctional facilities view attitude. Alternatively, social psychology tends to define attitudes as evaluations (Nunes et al., 2018), with the accepted 
definition of evaluations being "a psychological tendency that is expressed by evaluating a particular entity with some degree of favor or disfavor" (Eagly \& Chaiken, 1993, p. 1). Attitudes are also heavily focused on within social psychology in the context of sexual offending.

Cognitive distortions and attitudes are both used to study and examine sexual offending. However, Nunes and colleagues discuss in their 2018 study that it is possible that attitudes towards rape have tended to be lumped in with cognitive distortions about rape throughout the literature. This leads to some murkiness in the definition of not only attitudes and cognitive distortions as constructs, but also in the definition of evaluations in the context of sexual offending. It is true that evaluations and attitudes are thought of as one-in-the-same in social psychology, but perhaps evaluations are distinct in some ways as well.

This is not to say that evaluations are without a doubt their own separate construct, but it is possible that there are distinct elements of it that act as a subconstruct of attitudes - this is where evaluative attitudes become increasingly relevant. While Nunes and colleague's study examines rape against adult women, the results can be explored in the context of sexual offending against children as well. The implications of this study lead to the need to examine evaluative attitudes as a construct more closely.

The connection that evaluative attitudes may have with sexually aggressive behavior is exemplified by Hermann and colleagues (2018) who conducted a study that considered the relationship between explicit and implicit evaluations of sexual aggression and sexually aggressive behavior in both students and community men. Implicit evaluations are activated unintentionally, while explicit evaluations are intentional (Nunes et al., 2013). They hypothesized that those who have more positive explicit and implicit evaluations of sexual aggression would also have more self-reported past instances of sexual aggression, as well as a 
higher proclivity for sexual aggression. The results showed higher correlations between explicit evaluations of rape and sexual aggression, than implicit evaluations of rape and sexual aggression (Hermann et al., 2018).

The purpose of the current study is to examine evaluative attitudes towards sexual offending against children. Evaluative attitudes of sexual offending against children in this context encompass how negatively or positively one might think or feel about a sexual offensive act towards a child. For example, "you sexually touch or kiss a child who is deep asleep". Someone could either view this as a positive or negative thing. As will be discussed in the next section, there may be a distinct association between evaluative attitudes and sexually offensive behavior. If this is the case, the correctional field may be overlooking evaluative attitudes as an important factor in both assessment and treatment. While "'evaluative"" attitudes have not always been deliberately studied as their own construct in sexual offender literature, they may have manifested in scales that measure cognitive distortions and attitudes. Thus, if evaluative attitudes are distinct, they may be able to be found hidden amongst other scales.

As described in Hermann et al. (2018), evaluations are relevant in the study of sexually aggressive behavior. As such, there are several scales that had been developed originally meant to assess cognitive distortions or attitudes towards sexual offending towards children that may measure something beyond or other than those constructs - possibly evaluative attitudes. For example, a scale could have been meant to examine cognitive distortions, and the items of such scales may have also reflected cognitive distortions at first look. However, these items for cognitive distortions may have also taken on an evaluative quality while not explicitly denoting them as evaluative attitudes. Thus, it is possible that some of the already validated scales throughout the literature that intended to measure cognitive distortions could have also been 
inadvertently measuring evaluative attitudes. The same goes for scales intended to measure attitudes. This quality may be evident in the wording of the item. To avoid this pitfall and discern whether evaluations may be taking place on a scale or its individual items, I compiled a checklist as I analyzed each of the items in the scales of relevant studies to determine the extent to which evaluations may have been covered in the literature. It was also important to examine previous scales of sexual offending against children to gain inspiration for the structure and content of the items I developed for this study that are discussed later on.

The first step I used was: (1) to look at each item from the perspective of someone who thinks that sexual offending against children is either positive or negative (i.e., how would that individual answer the items?); (2) does the item directly ask the respondent how positive or negative the offending scenario described is (in other terms, how ambiguous is the item)?; and (3) whether the item uses positive or negative verbiage when describing an instance of sexual offending against a child (e.g., using words such as "better", "helpful", "affection", and "harm"). This checklist of potential evaluative qualities was not intended to provide a concrete conclusion as to whether an item is evaluative or not. Rather, it was intended to lay out an organized approach to assessing whether an item may or may not be evaluative or if the item is indicating an evaluation, amongst other things.

\section{Scales Potentially Assessing Evaluative Attitudes}

Abel and colleagues (1989) developed a scale that illustrated the relevance of cognitive distortions in those who have sexually offended against children. Their Cognition Scale contained 29 items with statements that represent common cognitive distortions about sexual offending against children (themes such as the child initiating behavior, minimization of harm, etc.). 
Participants were asked to rate their level of agreement towards a series of items in the form of statements on a Likert scale of 1 (strongly disagree) to 5 (strongly agree), with 3 representing a neutral option was selected. The items typically had some degree of mitigation in their wording, indicating that sexual offending against children may not be so bad. For instance, "Sometime in the future, society will realize that sex between a child and an adult is all right" (Abel et al., 1989, p. 151). Using the checklist I created, I assessed Abel and colleague's (1989) Cognition Scale. The following are a few examples of what I believed to be potentially evaluative items, and for comparison, some that may have been less evaluative, based on: (1) how someone who views sexual offending against children as a positive thing may look at the item, (2) the level of ambiguity of the item, and (3) whether there is the presence of any positive verbiage.

The first example is item 7: "Having sex with a child is a good way to teach the child about sex" (Abel et al., 1989, p. 150). If someone felt strongly that sex with a child was a positive thing, they may be inclined to think that this item was acceptable, but they may not think it is the best way to teach a child about sex, functionally. Inversely, if someone felt strongly that sex with a child was a negative thing, they may not feel that this is the right thing to do to teach a child about sex. However, they may agree it would be functionally the right thing to do, as in, it would be effective in the instruction of sex. According to the first point on my checklist, this item did not seem to be initially evaluative, as there are too many ways it can be interpreted by both someone who thinks sex with children is good or someone who thinks it is bad. Regarding the second point on the checklist, the item did not directly ask if the statement is positive or negative, nor did the response scale imply evaluation; the participant would either agree or disagree with the item, and this does not indicate levels of positivity towards the item. This item was ultimately more ambiguous than some of the others that will be discussed, which reduces the likelihood of it 
reflecting an evaluation. Despite the ambiguity of the item, the material of the item itself was consistent with positive evaluations given the verbiage of "a good way". This item only met one of the three potential indicators of an evaluation, so it may not have really been measuring evaluations at all.

Another example was item 15: "I show my love and affection to a child by having sex with her (him)" (Abel et al., 1989, p. 151). If an individual held positive evaluations towards sexual offending against children, they might agree with this statement. Previous literature has suggested that a common reason that men give for sexual offending against children is to show love and affection (Janke et al., 2018). This could have indicated an evaluation. Showing something love typically indicates favoring it or viewing it as a positive thing. This item was somewhat as ambiguous as the last item; however, it was less ambiguous in that it illustrates someone (i.e., I) shows their love by sexually offending against a child, as opposed to a general statement of some unnamed person sexually offending against a child. The ownership implied by the action of the item leads the respondent to put themselves in that situation and decide if they would also show love and affection that way. The most evaluative element of the item was the positive verbiage "love" and "affection." As such, item 15 may have been more evaluative than item 7.

By my criteria, one of the least evaluative items in the scale was item 1, "If a young child stares at my genitals it means the child likes what she (he) sees and is enjoying watching my genitals." (Abel et al., 1989, p. 150). This item was more clearly a statement of assumption than an evaluation. This item held the same logic of the following hypothetical statement, "That child must be sad, because they are quiet". If someone who held positive evaluations of sexual offending against children read this, it is possible they could disagree; maybe this child does not like the hypothetical person's genitals, but it is still ok to have sex with them. However, if someone who 
had negative evaluations of sexual offending against children, they could agree that the child may be interested in the adult's genitals, but the idea of the adult having sex with the child is negative. Based on the different perspectives that a respondent could have taken answering this item, ambiguity was displayed enough to indicate that the item may not be getting at evaluations at all. While the item did have slight positive verbiage (e.g., "like"), it was about what the child may like, not whether the action is positive or negative. It is more likely that this item reflected a cognitive distortion.

In 1996, Bumby sought to refine Abel's original measure of cognitive distortions towards sexual offending against children. While Abel's scale was thought to have reasonably reliable psychometric properties, there was still room for improvement. Notably, two items addressed the treatment of the offender, as opposed to the cognitions of the offender (Bumby, 1996). Bumby developed two versions of a revised cognitive distortions scale: The Rape Scale and The Molest Scale (Bumby, 1996), which addressed adult female victims and child victims, respectively. The Molest Scale was the most relevant to this study; therefore, the discussion will be confined to that scale.

The Molest Scale consisted of 38 items that depict sexual offending against children. The Likert scale is four-points ranging from 1 (strongly disagree) to 4 (strongly agree). Like Abel's scale, there were a few items that were potentially evaluative. Item 1 stated, "I believe that sex with children can make the child feel closer to adults" (Bumby, 1996, p. 51). If an individual were to have a positive evaluation of sexual offending against children, it is possible they would strongly agree. However, it is possible for someone who has a positive evaluation to disagree because they know it is not good for the child; they just do not care. This item was less ambiguous than the items discussed in The Cognitions Scale because it explicitly asked the 
respondent to rate the extent to which they think a sexual offence against a child can have a positive outcome (not in the response scale itself, but in the direction of the question). However, this item could have been less an evaluation of the sexual offence itself and more of the aftereffects. It should also be noted that the item contains positive verbiage such as "closer" in the context of forming relationships and bonds. This item may have been evaluative, but perhaps not directly of the offence.

In comparison, item 29 illustrated an altogether seemingly non-evaluative statement. "If a person tells himself he will never molest again, then he probably won't”' (Bumby, 1996, p. 51). This item seemed to be less subjective than item 1. Not everyone would agree with this statement; however, it does not focus on the actual act of sex with a child and whether it is more tangential. If someone were to have a positive evaluation of sexual offending against children, they could either agree or disagree with this. Hypothetical willpower has no clear connection to whether the action is good or bad. The same can be said for someone with a negative evaluation of sexual offending against children. This item also did not lead the respondent to contemplate if they think the action could be good or bad; it simply states that if someone did not want to sexually offend a child, then they would not. For the same line of reasoning, this item was more ambiguous than some of the others. This item also did not contain any positive or negative verbiage.

Later, Jahnke, and colleagues (2018) tested a scale that measured, as they defined them, "moral attitudes" towards sexual offending against children. In this study, the word "moral" described a seemingly copasetic scenario of a sexual offence against a child in which there was no perceived harm towards the child (sexual, physical, mental, or emotional) within the item. The study assessed moral attitudes towards sexual offending against children in a sample of 
community men in Germany who were sexually attracted to children (pedophilic), comparing their scores to a group of non-pedophilic men. The Moral Attitudes Scale (Jahnke et al., 2018) rated levels of direct and indirect harm, no consent, exploitation, and differences in adult and child sexualities that were observable in each item (Jahnke et al., 2018). The hypothesis was that if the pedophilic participants perceived a child as appearing to enjoy, or at least consent to sexual relations with an adult man, that they would have more accepting attitudes towards the behavior than their non-pedophilic counterparts (Jahnke et al., 2018). Participant's opinions of moral rightness/wrongness in each category were assessed when in the context of a seemingly agreeable sexual situation (e.g., the child smiling, exclaiming that they enjoyed the contact, etc.). The following are a few examples of different moral themes in the items:

- direct harm, "The sexual contact was beneficial for the child's development" (Jahnke et al., 2018, p. 932)

- indirect harm, "The child will be stigmatized if others find out about the sexual contact" (Jahnke et al., 2018, p. 932)

- no consent, "The child was probably in over his or her head when confronted with the adult's sexual wishes and needs" (Jahnke et al., 2018, p. 932).

- exploitation, "The adult was more powerful than the child, which made it impossible to have an equal sexual relationship" (Jahnke et al., 2018, p. 932)

- the incompatibility of child and adult sexualities, "The child had his or her desires met in the relationship with the adult" (Jahnke et al., 2018, p. 932) Participants indicated whether they agreed with the item by circling "yes" or "no" next to each question. These items tended to mimic cognitive distortions despite being labeled as "moral attitudes" and may potentially reflect evaluative attitudes. It could be argued that viewing sexual 
offending against children as being acceptable if an adult means well, or if the child enjoys it, is not the same as evaluating the action as good or bad. However, while these items did not appear to explicitly show an evaluative nature of good or bad, according to Jahnke and colleagues, there is an understanding amongst pedophilic men that "child-rape"(i.e., non-consensual adult-child sex) is inherently bad (Jahnke et al., 2018). Thus, these items of the Moral Attitudes Scale may also have reflected evaluations.

For example, item 10 read, "The sexual contact probably deepened the bond between the adult and the child" (Jahnke et al., 2018, p. 932). This item is like item 1 in Bumby's MOLEST scale, "I believe that sex with children can make the child feel closer to adults" (Bumby, 1996, p. 51). The same checklist results for Bumby's item 1 can be applied to Jahnke and colleague's item 10. Item 16 on the Moral Attitudes Scale states, "This sexual contact was beneficial for the child's development" (Jahnke et al., 2018, p. 932). Someone with a positive evaluation of sexual offending against children may agree or disagree, and the same goes for if someone held a negative evaluation. This item was ambiguous because someone could have thought that the action was bad but has good outcomes or vice versa. This item suggested a positive outcome due to the encounter, and the positive verbiage of "beneficial" indicates an evaluation could have been taking place. Compared to some of the other items, this one may have reflected evaluations a bit more.

An example of a less evaluative item was item 14, "Consciously or subconsciously, the adult used the child for his own pleasure and interests" (Jahnke et al., 2018, p. 932). This item was like The Molest Scale item 29, "If a person tells himself he won't molest again, he probably won't" (Bumby, 1996, p. 51). These items both paid more attention to the actions and intentions of the respondent, as opposed to offering a scenario with the child is actively involved. This 
takes the focus off the direct action of a sexual offence. This item was more ambiguous than some of the others because it really has nothing to do with how bad or good sexual offending against children is. This item was more concerned about the offender motive. This item also used neutral verbiage. An individual's response to this item potentially had little to do with their evaluations of child sexual offending. The extent to which evaluative attitudes were present in this scale was somewhat more ambiguous than the other scales discussed. However, Jahnke and colleague's scale had an evaluative undertone overall, considering the implicit idea that "childrape" was bad amongst the scale's pedophilic respondents.

The examples provided from the previous scales indicate that, historically, evaluative attitudes may have not been of primary concern in the sexual offender literature. It is arguable, however, that evaluative attitudes were reflected in some of the items. A more explicit spotlight should be shone on evaluative attitudes to examine to what, if any, extent they may be relevant amongst sexual offenders against children, which leads to the current study.

\section{The Current Study}

The current study assessed the extent to which evaluative attitudes towards sexual offending against children may play a role in sexual offending against children. I used a series of items that I created based on themes and wording from the scales I assessed in the previous section and from other studies by Nunes and colleague's that best reflect potential evaluative attitudes. There were two goals for this study: (1) identify items in the pool I have created with the highest variability amongst participants and (2) to see if what I have created to reflect evaluative attitudes towards sexual offending against children has any connection to sexual offending behavior with my sample of incarcerated men. 


\section{Method}

\section{Participants}

A total of 188 participants responded but was reduced to 172 by removing cases of those who had failed the attention check, those with missing data for item responses, and those who did not indicate offence history. The participants were all male, except for 11 who did not list their gender, and two who responded as "other". The average age of the participants was 40years-old and ranged from 22-years-old to 72-years-old. The racial composition of the participants was predominately white (79.1\%). Participants were asked to report their current and prior offences, see table 1 for a more detailed demographic breakdown.

Participants were grouped for analysis based on their offences, categorized by those with current, and-son sexual offences against children (SOC) and those without current, hands on sexual offences against children (Non SOC). Those with both a current sexual offence against a child 13-years-old to 15-years-old, and 12 or younger, and a child pornography offence were included in the SOC group. If the participant only had an offence against a 13-years-old to 15years-old and no offence against a child 12 or under, or only a child pornography offence with no offence against a child 12 or younger, or only prior sexual offences against a child of the age range mentioned above, they were excluded from the comparison groups all together. Due to this, the $n$ for comparison groups is actually 83 , instead of the original number of participant cases used to refine the items (172). This was done to get the sample sizes for each group as close together as possible. Participants with all other current or prior offences were in the Non SOC group. 
Table 1

Participant Demographics

\begin{tabular}{lc}
\hline Ethnicity & $\%(n)$ \\
\hline White & $79.1 \%(136)$ \\
Black & $15.7 \%(25)$ \\
Indigenous & $2.3 \%(4)$ \\
Hispanic & $1.7 \%(3)$ \\
Arab & - \\
East Indian & - \\
Missing & $2.3 \%(4)$ \\
\hline Offence (Current) & $85.4 \%(147)$ \\
SOC & $12.2 \%(21)$ \\
Non SOC & $.6 \%(1)$ \\
Missing & $1.7 \%(3)$ \\
\hline Offence (Prior) & \\
SOC & \\
\hline Non SOC & \\
\hline
\end{tabular}




\section{Item Development}

\section{Item Questionnaire}

The item questionnaire contained 60 items (and two quality control questions) designed to measure evaluative attitudes toward sexual offending against children 13 years old or younger in males with a history of both sexual and nonsexual offending. These items were developed for the current study (see appendix A for the full list of items). Development of these items began with a larger pool of items, which were reduced and revised based on feedback from students in Dr. Nunes's lab and from colleagues who conduct research and clinical work with people who have sexually offended against children.

The development and structure of the measure were inspired by Nunes and colleagues (2021) Evaluation of Violence Questionnaire (EVQ). The MOLEST scale was helpful for inspiring item content themes and potentially mitigating factors that I could incorporate. For example, item 1 on the MOLEST scale reads, "I believe that sex with children can make the child feel closer to adults" (Bumby, 1996, p. 51). The mitigating factor is the suggestion that the sexual offence was benevolent to the child and meant to help them.

Mitigation was important to consider in crafting these items to increase the likelihood of item variability. Item variability was important for avoiding highly skewed or unbalanced items (commonly referred to as a floor effect). If all 60 items lacked any variability at all, this would have indicated that everyone had the exact same response to the items and none of them would have been useful for studying evaluative attitudes towards sexual offending against children (Clark \& Watson, 2019). Item 1 The Molest Scale described earlier had a potentially mitigating element; the idea that the act of sex with a child in some way is helpful to the child. Other examples of mitigating themes included the child initiating sexual contact and excusing the adult 
for initiating sexual contact due to an underlying issue the adult is suffering from, such as stress or depression (Bumby, 1996).

To maximize variance, sexual contact was described as abstractly as possible while still avoiding ambiguity. To be clear, the role of avoiding ambiguity for these items is to ensure each item clearly illustrates a sexual offence. For the sake of homogeneity and a manageable scope of the study, each item described contact offences only. Each item described sexual offending in a variety of scenarios: when the child is asleep, helping the child put on a bathing suit, etc. The questionnaires were put into four versions, each version in a different fixed randomized order to control for potential order effects. Participants responded to each item using either the Likert scale of 1-4, (1) "very bad", (2) "pretty bad", (3) "not so bad”, (4) "not bad at all" and then (1) "very negative", (2) "pretty negative", (3) "not so negative" (4) "not negative at all", or the other way around. This semantic differential scale was used because similar studies examining attitudes and evaluative attitudes used this format as well, and semantic differential scales have been widely used and validated for measures looking at evaluative attitudes within social psychological research for a while (Nunes et al., 2015, 2018). For the purposes of this study, two different wording styles (bad/negative) were used to optimize response variability, and additionally to thoroughly test each item twice with less risk of participant fatigue or boredom through looking at the same response scale each time through. The response scales provided only indicated levels of negativity or badness (as opposed to having the option to rate an item as "positive" or "good") because when dealing with the topic of sexual offending against children, it is important to endeavor to avoid making the respondent feel like they are being accused of feeling positively about something deemed generally bad within society. 


\section{Additional Measures}

\section{Demographic Questionnaire}

A demographic questionnaire was developed for the current study to gather information about participant demographic and offence history (see Appendix B for the full questionnaire). Questions included demographic questions such as age, gender, and ethnicity. Offence questions included current and previous offences that they have been convicted of. The pre-questionnaire was developed to obtain offence information, as I cannot access individual case files; this would break anonymity. Offence history was important because it informed the comparison groups.

\section{Procedure}

I was granted permission from the Missouri Sex Offender Program (MSOP) to gain access to their inmates as participants. Upon getting approval from the Carleton University Research Ethics Board (CUREB), I was granted formal permission from the institution to give the opportunity to their clients to participate in the study.

A clinical psychologist who manages the MSOP recruited participants and administered the questionnaires. Participants decided whether they would complete the materials by indicating their consent (or lack of consent) via implied consent (see appendix C). Participants were instructed to not include their name or any other indicator of personal identification, regardless of if they finished the study. Participants were then given a debriefing form (see appendix D) and were directed to a staff member if they experienced any emotional or psychological distress from the questionnaire material.

\section{Statistical Analysis}

Item variation was first examined to decide which items should be refined for the main analysis. An independent-samples $t$-test was then used to examine the relationship between the 
two offence groups: the first group, SOC, includes all participants who have a current contact sexual offence against a child 12-years-old or younger, while excluding individuals who only had a prior sexual offence against a child 12-years-old or younger, or only a child pornography offence. The comparison group included all those without any sexual offences against children. Regarding the age of sexual offence victims, even though the survey details that a "child" is 13 or younger, for analysis purposes, I had to reduce the SOC group to just 12 and younger to get sample sizes that were more equal in size. I ultimately made the decision to not more finely define the offence into more than two groups, because I wanted this study to act as a baseline for examining how my items performed with the basic SOC vs Non SOC group. This is addressed further in the discussion section. Finally, aside from statistical significance, effect size was evaluated.

\section{Results}

\section{Item Reduction}

The first part of my analysis required that I reduce and refine the initial list of 60 items that depicted sexual offences against children. To do this, I merged each version of the items to a master list of 60 . I then sorted the items by variance and took the 15 items with the highest variance. These reduced items will be referred to as Evaluative Attitudes towards Sexual Offending against Children (EASOC). Overall, the variance of each item in the EASOC ranged from 0.206 to 0.749 . Table 2 shows the new refined items for the main analysis, and Table 3 shows the descriptive statistics of the remaining items. It should be noted that for the remaining items, there will be two versions of each item (one with the "bad" response scale and one with the "negative" response scale). The EASOC only had one version of each item selected in order to retain only the items with the highest variance. 
Table 2

EASOC Descriptives

Item

Mean Std. Deviation Variance

Your partner wants to sexually touch a child with you, so you do it to make your partner happy $(\mathrm{N})$

1.18

0.86

0.74

A child wants to touch your penis, and you let them

for your sexual pleasure $(\mathrm{N})$

$\begin{array}{lll}1.16 & 0.54 & 0.29\end{array}$

You are bathing a child and they ask you to wash their private parts. You wash and continue to rub their

1.16

0.52

0.27

genitals to make the child feel good (B)

You let a child touch you penis for their sexual

1.18

0.52

0.27

pleasure (B)

A child asks you about masturbation so you

1.15

0.51

0.26

masturbate in front of the child to teach them how to

do it (B)

A child asks you about masturbation so you touch their genitals to teach them how to masturbate (B)

A child puts their hand on your penis, and you let it continue for your sexual pleasure $(\mathrm{N})$

While you're drunk, you sexually touch a child (N)

1.15

0.49

0.24

1.15

0.48

0.23

You have an erection as you hug a child. You continue

1.15

0.48

0.23

to hug the child, pressing your penis against the child

$1.13 \quad 0.48$

0.23

(B)

You sexually touch a child who doesn't have a lot of friends so that they can enjoy some attention (N)

To prepare the child for the adult world, you get two

0.47

0.22

children to sexually touch each other while you watch (N)

You sexually touch a child who is sleeping deeply (B)

1.12

0.47

0.22

A child asks you what a penis is, so you show them yours and have them touch it for your sexual pleasure (B)

$\begin{array}{lll}1.11 & 0.46 & 0.22\end{array}$

A child sits on your lap and rubs against your penis,

1.13

0.46

0.21 you let it continue for your sexual pleasure $(\mathrm{N})$

You have a tickle fight with a child and touch their

1.12

0.45

0.20 genitals for your sexual pleasure (B) 
Note. Response scale of either "very bad" or "very negative" denoted in items by either (B) or $(\mathrm{N})$, respectively.

Table 3

Remaining Item Descriptives

Item

Mean Std. Deviation Variance

A child asks you about masturbation so you touch their genitals to teach them how to masturbate $(\mathrm{N})$

$\begin{array}{lll}1.12 & 0.45 & 0.20\end{array}$

A child asks you about masturbation so you masturbate in front of the child to teach them how to do it $(\mathrm{N})$

0.45

0.20

A child sits on your lap and rubs against your penis, you

let it continue for your sexual pleasure (B)

$\begin{array}{lll}1.13 & 0.45 & 0.20\end{array}$

A child asks you about sex, so you sexually touch the child to teach them $(\mathrm{N})$

$\begin{array}{lll}1.13 & 0.45 & 0.20\end{array}$

To prepare them for the adult world, you get two children to sexually touch each other while you watch (B)

$\begin{array}{lll}1.12 & 0.44 & 0.20\end{array}$

You sexually touch a child to help them feel better about their body $(\mathrm{N})$

$\begin{array}{lll}1.12 & 0.44 & 0.20\end{array}$

You sexually touch a child who is passed out $(\mathrm{N})$

$\begin{array}{lll}1.11 & 0.44 & 0.19\end{array}$

Your romantic partner doesn't want to have sex with you, so you sexually touch a child instead (B)

0.44

You are helping a child into their bathing suit, and you touch their genitals for your sexual pleasure (B)

$1.14-0.44$

0.19

You sexually touch a child who you know has been neglected or abused, to show them love $(\mathrm{N})$

$\begin{array}{lll}1.12 & 0.43 & 0.19\end{array}$

While you're drunk, you sexually touch a child (B)

$\begin{array}{lll}1.11 & 0.43 & 0.18\end{array}$

You are lonely, so you sexually touch a child to feel close to someone (B)

$\begin{array}{lll}1.11 & 0.43 \quad 0.18\end{array}$

A child asks you what a penis is, so you show them yours and have them touch it for your sexual pleasure (N)

$\begin{array}{lll}1.11 & 0.43 & 0.18\end{array}$

You sexually touch a child who you know has been neglected or abused, to show them love (B)

0.42

0.18

A child asks you about sex, so you sexually touch the child to teach them (B)

$\begin{array}{lll}1.11 & 0.42 & 0.18\end{array}$

You sexually touch a child who doesn't have a lot of friends so that they can enjoy some attention (B)

$1.11 \quad 0.42$

0.18 
You sexually touch a child who is sleeping deeply (N)

1.10

0.41

0.17

Your partner wants to sexually touch a child with you, so you do it to make your partner happy (B)

$\begin{array}{lll}1.12 & 0.41 & 0.17\end{array}$

You sexually touch a child because their parents are

neglecting them, and you want them to feel loved $(\mathrm{N})$

$\begin{array}{lll}1.09 & 0.41 & 0.17\end{array}$

You have a tickle fight with a child and touch their

genitals for your sexual pleasure $(\mathrm{N})$

$\begin{array}{lll}1.11 & 0.41 & 0.17\end{array}$

You are helping a child into their bathing suit, and you

touch their genitals for your sexual pleasure $(\mathrm{N})$

$\begin{array}{lll}1.12 & 0.40 \quad 0.16\end{array}$

You sexually touch a child who is passed out (B)

$\begin{array}{lll}1.10 & 0.40 \quad 0.16\end{array}$

You are very depressed, so you sexually touch a child to try to feel better $(\mathrm{N})$

$\begin{array}{lll}1.11 & 0.39 & 0.15\end{array}$

You are so horny that you sexually touch a child who is close by $(\mathrm{N})$

$\begin{array}{lll}1.10 & 0.39 & 0.15\end{array}$

You sexually touch a child because they won't reject you like adults will $(\mathrm{N})$

$\begin{array}{lll}1.09 & 0.38 & 0.14\end{array}$

You sexually touch a child (B)

$\begin{array}{lll}1.11 & 0.38 & 0.14\end{array}$

You sexually touch a child to help them be less shy (B)

1.08

0.38

0.14

You sexually touch a child $(\mathrm{N})$

1.09

0.37

0.14

You tuck a child into bed at night and touch them

sexually to help them get to sleep (B)

1.08

0.37

0.13

You sexually touch a child to help them be less shy $(\mathrm{N})$

1.08

0.37

0.13

You are lonely, so you sexually touch a child to feel

close to someone $(\mathrm{N})$

$\begin{array}{lll}1.11 & 0.37 & 0.13\end{array}$

You are very depressed, so you sexually touch a child to try to feel better (B)

0.36

0.13

You sexually touch a child because their parents are neglecting them, and you want them to feel loved (B)

$\begin{array}{lll}1.08 & 0.36 & 0.13\end{array}$

Your romantic partner doesn't want to have sex with you, so you sexually touch a child instead $(\mathrm{N})$

$\begin{array}{lll}1.08 & 0.35 & 0.12\end{array}$

You sexually touch a child to help them feel better about their body (B)

$\begin{array}{lll}1.09 & 0.35 & 0.12\end{array}$

You sexually touch a child because they won't reject you like adults will (B)

You see that a child is sad, so you sexually touch them to

$\begin{array}{lll}1.09 & 0.34 & 0.12\end{array}$

You are so horny that you sexually touch a child who is close by (B)

A child asks you about masturbation so you touch their genitals to teach them how to masturbate $(\mathrm{N})$

$\begin{array}{lll}1.12 & 0.45 & 0.20\end{array}$


A child asks you about masturbation so you masturbate in front of the child to teach them how to do it (N)

A child sits on your lap and rubs against your penis, you let it continue for your sexual pleasure (B)

Note. Response scale of either "very bad" or "very negative" denoted in items by either (B) or (N), respectively.

\section{Group Comparison}

The second portion of my analysis compared the total score means of two offence groups:

(1) those with either current and prior, or current-only, sexual offences against children (12-years old and younger), and (2) all other offences. The 62 participants with current contact sexual offences against children $(M=1.159, S D=0.389)$ compared to the 21 participants without any sexual offences against children $(M=1.000, S D=0.000)$ scored significantly higher on the EASOC, with $t(61)=3.217, p=.002$. In other words, those with current and prior sexual offences against children rated the items depicting sexual offending than as significantly less negative did those with other offences or sexual offences against adults. The effect size for this analysis is moderate $(d=.471)$. This relationship is illustrated in Figure 1. 
Figure 1

Comparison of Mean Scores on the EASOC Between SOC and Non SOC

EASOC means between SOC and Non Soc

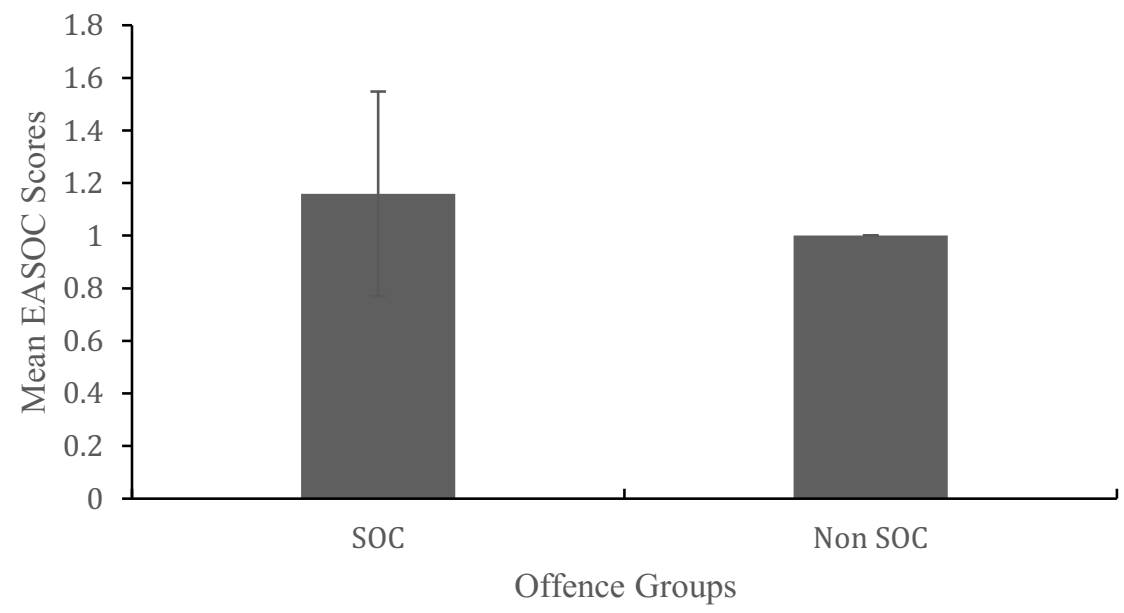




\section{Discussion}

The goal of the current study was to develop a measure that may reflect evaluative attitudes towards sexual offending against children. The analysis process was two-fold: (1) reduce the 60 items I created to a list of 15 items with the highest variation amongst respondents and (2) see if there is a relationship between offence groups and mean scores from the EASOC.

The results indicated that there was a significant relationship between offence history and the mean score of responses to the EASOC I developed. Participants who had current and handson sexual offences against children had higher mean score on the EASOC, and participants who did not have current and hands-on sexual offences against children had a lower mean score on the EASOC. Those with a higher mean score indicates that they rated sexual offending against children as less negative, and those with a lower mean score rated sexual offending against children as more negative. These results came with a medium effect size of .471, which indicates that the significant relationship is noteworthy, and should be further examined.

The results indicate that there may be a significant relationship between evaluative attitudes and sexual offending against children. An alternative interpretation is that respondents answered in a way that indicated how they felt intellectually about sexual offending against children, but not from a place of evaluative attitude, or attitude in any way. In other words, respondents could have known that the societal standard is to view sexual offending against children as negative (especially those who have been incarcerated for it) but they do not truly feel that it is bad. If this is the case, either the items I developed are not evaluative in nature, or the issue lays in encouraging the participant to be truthful and actually "“'evaluate"' the items, as opposed to just answering the way they think they ought to. The issues that come with self-report surveys and respondent truthfulness will be discussed in the limitations section. 
Something interesting to consider along the lines alternative explanations for the results, is that is it possible that the EASOC items actually are more illustrative of cognitive distortions than evaluative attitudes. When thinking critically about how someone may respond to my items, it is arguable that they would inherently need to possess some form of cognitive distortion in their thought process to view the items as anything other than bad or negative. As discussed in the literature review, the definition of evaluative attitudes is a bit murky, and it is possible they fall under the umbrella of cognitive distortions as a subconstruct. Where the EASOC items are distinct from cognitive distortions, however, is that they are not necessarily "exaggerated" or “irrational" as defined by Beck (1979). For example, "You sexually touch a child who is sleeping deeply" is not exaggerated or irrational, but if someone were to think this is not bad at all, it is possible they have underlying disordered ideas of what is acceptable. Beck's description of cognitive distortions may also be inaccurate or dated, as it was formed several decades ago.

As discussed in the review of the literature, it is generally found that attitudes are relevant to a history of sexual offending in adults (Hermann at al., 2018; Nunes et al., 2018). It was also discussed that attitudes are relevant in those who may not have had a contact offence against a child but did view sexual contact with children as something other than negative (Jahnke, 2018). The current study is the first to explicitly look at the possible relationship between evaluative attitudes and their connection to sexual offending against children. While the scale I developed is not validated, the ideal purpose for it is to fill in the gaps sexual offender literature in the context of sexual offending against children. With the relevance of attitudes that has been discussed in the literature, and the findings that evaluative attitudes may be distinct from attitudes and cognitive distortions, and an important factor in sexual offending, it is important that research extends itself to sexual offending against children. Regarding the implications of the results of 
this study on on sexual offender treatment, while the EASOC is still in early days, it will likely lend itself as a very useful tool in the future. Self-report measures are often used in assessing and treating sexual offenders against children, adults, and in the correctional domain as a whole. My development of the EASOC might supplement other scales that measure cognitive distortions and other types of attitudes. In other words, the EASOC might someday assist in creating a more well-rounded means of assessment of sexual offenders against children in the correctional domain. The implications of the study as whole were discussed above, and now it is time to discuss the elements of the EASOC in a more detailed way.

\section{Components of the Reduced Items}

There was a large emphasis on mitigating factors in the creation of my items for this study. This was done not only for an ideal variation on responses but also to make the items as evaluative as possible. When I reduced my final items based on the top 15 highest response variances (indicating that not everyone just responded "very bad" or "very negative"), there were some important mitigating elements to observe. The mitigation in the items reduced fell into a few different categories. For the following, it should be assumed that each category is perceived, and not a universal truth. For example, an item mitigating factor might be the perception of being helpful to the child - arguably, most people would not think this way. Interestingly, amongst the 60 items I reduced to 15, the "bad" response scale appeared more than the "negative" response scale in the final list.

The first type of mitigating circumstance that can be observed is accidental touching. For example, an item with one of the highest response variances was item 56, "A child sits on your lap and rubs against your penis, you let it continue for your sexual pleasure". The second type of mitigating circumstance was the perception of being helpful to the child. Item 9 is a perfect 
illustration of this, "A child asks you about masturbation, so you touch their genitals to teach them how to masturbate". The third type of mitigation I observed was what I refer to as unconscious circumstances (i.e., the child is asleep or the participant in the scenario is intoxicated, as in item 3, "While you're drunk, you sexually touch a child").

The fourth and final mitigating theme I observed for my reduced items is child initiation, item 7, "you let a child touch your penis for their sexual pleasure". Each of these four mitigating themes appears more than once in the reduced list of 15 items, and it indicates that the specific scenarios surrounding each item encouraged more critical thought when responding, and perhaps deeper evaluation.

The logic of a potentially mitigating circumstance allowing for a less negative evaluation of any offensive sexual act against a child ties in with my previous examination of the study regarding moral attitudes and sexual offending against children (Jahnke et al., 2018). Jahnke and colleagues found that the majority of their participants who were self-reported pedophiles had a somewhat permissive attitude towards sexual offending against children, if the child appeared to enjoy it. While my study does not address those with pedophilia, and the Jahnke study does not explicitly address evaluative attitudes, these findings may be similar to my ideas regarding mitigating factors of an offence having an effect on feelings towards that offence.

\section{SOC vs Non SOC: Comparing Mean Score Responses}

In the current study, those with sexual offences against children scored significantly higher on my items (see Table 2) than those without sexual offences against children. This outcome was what I had originally hypothesized, and it appears to correspond with the overall trend in similar literature - that those who have sexually offended or exhibited sexually aggressive behavior will likely not view sexually offensive behavior as negatively as those without such a history (Nunes et al., 2013; Widman \& Olson, 2013; Hermann et al., 2018; Nunes 
et al., 2018). However intuitive this trend might be, it is important to also consider the extent of the relationship. Therefore I draw attention to the effect size in my analysis and not just the significance. Effect size is useful when dealing with new or unknown scales, or the preliminary items to scales (Coe, 2001), as present in the current study. Although I had significant group comparisons, the effect size was moderate $(d=.485)$, given that a large effect size is typically $d$ $=.8$ or higher (Coe, 2001). This could imply a few things. First, it is possible that there are factors not examined that would have been applicable to my study and made for a stronger effect, such as participant education levels, sexual orientation, or intellectual capacity. A moderate effect size is not bad, but it may imply the possibility that even if my items successfully represented evaluative attitudes and were validated, that evaluative attitudes are not the strongest construct to use in examining sexual offenders against children. The latter of these two options feels unlikely; however, as mentioned earlier, there is an overall trend that sexually aggressive behavior is linked to less negative attitudes towards sexual offending, so the same could be true for sexual offending against children. The possibility that there are other relevant demographics to observe in participants will be considered when discussing the limitations of this study.

While every effort was made to ensure an effective and accurate study, there were a few limitations that should be taken into consideration. As previously mentioned, data were collected during the COVID-19 pandemic, as such, access to participants was severely limited. The study utilized data from participants within the Missouri Sex Offender Corrections program; however, there were several other institutions across the US and Canada that had shown interest in participating prior to COVID-19. Another limitation is that the items were created specifically for this study and are not from a validated measure. Thus, there is no way to tell what construct the items are truly reflecting or how precise those reflections are. However, the items were based 
on other validated scales, which provides a hopeful outcome of moderate to strong validity when future studies seek to validate the refined items.

Furthermore, participant demographic information other than the items about offence history were not examined in this study. For example, there was no recording of potentially important variables, such as sexual orientation, intellectual capacity, or education. This was avoided to keep the scope of the study narrow and to focus on those with sexual offences against children and those without. It remains possible that variables such as sexual orientation or intellectual capacity could have been confounding in the exploration of evaluations.

On a similar note, it must be acknowledged that during the current social climate, racial demographics are essential to collect and consider with research moving forward. However, the current study was intended to act as a baseline for the EASOC and the goal was to get as clear of an idea as possible on how the items performed with SOC vs Non SOC groups. It is also important to point out that since the sample size was severely limited due to the pandemic, the study prioritized SOC vs Non SOC for creating comparison groups to keep in line with the original study question.

There are a few issues consistent with self-report surveys that could have hindered item response. It has been noted previously that participants in studies regarding sexual offending, despite anonymity, are reluctant to fully disclose their true feelings surrounding offensive situations (Nunes et al., 2013). The items depicted somewhat graphic situations of sexual offending against children, so it would be reasonable for participants to feel inclined to respond in a way that indicates they felt all situations were as bad as possible. Another factor that could have altered responses amongst the participants was the fear of being identified. Despite making every effort to ensure anonymity and to reassure participants of anonymity, some could have still 
altered their answers for fear of being linked to their responses. This is not likely; however, the items all had upsetting material, and the caution of the participants may have shown in the responses.

In the same vein, it is possible that another limitation could have been inaccurate offence reporting. Participants may have not reported sexual offences against children, listing their offence as against adults instead. This is a limitation because if anyone were dishonest, that would make it increasingly difficult to determine not only if EASOC items were truly reflecting evaluative attitudes, but also if there was a significant connection to the items and offence history as the results originally indicated. There is really no way to know if this is happening, however a potential way to reduce effects this possibility is to test the EASOC with as many samples from as many different backgrounds (community, university, etc..) as possible. Inaccurate offence reporting could be a result of social desirability, however, considering that this current participant pool was all from the same sex offender program and everyone was anonymous, it is not likely. Where social desirability may become more of an issue is when researchers test the EASOC with samples of community or university men, since they typically are not in an environment where everyone has committed a crime of some kind.

Another limitation of this study is that non-probabilistic sampling was used. This was not a random sample of the population; rather, it was a curated sample from one location broken down into known groups (sexual offenders against children, sexual offenders against adults, all others). Thus, these results may not be generalizable to the population. After validating the refined items, future studies ought to utilize a random sample from the community, or other populations of interest such as a larger sample of men with convictions of a sexual offence against a child. 
I endeavored at several points in the study to compensate for these limitations. During the creation of the items, Dr. Nunes and I went through several drafts of the list to have ideal wording. We also sent each item draft out to our lab for review and to several of Dr. Nunes's professional colleagues. The items were designed to be clinical and straightforward, however not so clinical that the participants could not relate to the situations (e.g., the use of the phrase "horny" and other colloquial terms). During the actual study, it was emphasized in the instructions and the debriefing form that this was entirely anonymous.

Moving forward, future researchers may want to consider several things for replicating and advancing this study. For instance, a larger sample size would behoove generalizability, especially in a community sample. Most prominently, future study should take place during a less chaotic time in the world (i.e., COVID-19 global pandemic); although, that is less easy to control.

The creation of an implicit association test (IAT) for sexual offending against children may be interesting to develop. An IAT may help reduce social desirability effects (particularly in community and university samples) because participants will not be explicitly asked to evaluate items that depict sexual offending against children, so they may be less defensive. A design similar to the rape evaluation IAT used in Hermann and colleagues study looking at implicit and explicit evaluations of sexual aggression in community men (Hermann et al., 2018) may be a good place to base the design from. The IAT developed for sexual offending against children could then be compared to the EASOC for further insight into evaluative attitudes and where implicit and explicit evaluations fall into place.

Regarding the study design, future researchers may want to look into more finely separating the offence groups into three comparison groups if they have a large enough sample. 
The current study stuck to two groups because a) I felt it was important to establish a base line of item performance for the EASOC with the simple SOC vs Non SOC, and b) the sample size I

was working with was pretty limited due to COVID. However, future studies may be successful with gathering data from multiple institutions (yielding a much larger sample size) and perhaps breaking the comparison groups down into four categories: offenders against children aged 1216, offenders against children 11 and under, offenders against adults, and all other offenders. Future research may also consider working on construct validity by testing the EASOC with as many different groups of men as possible in hopes of achieving some generalizability with results.

\section{Conclusion}

In summation, participants incarcerated for current, hands-on sexual offending against children had a significantly higher mean score on the EASOC than did the participants without current, hands-on sexual offences against children. In other words, this implies that the participants with the current hands-on offences may have a less negative view of sexual offending against children, than those who did not. The word "iimplies"" is critical because the items I created have not been validated, and it is not truly known whether they truly reflect evaluative attitudes. Given that previous literature suggests a connection between evaluative attitudes and sexually aggressive behavior in adults, this study opens a dialogue for continuing research of this connection regarding sexual offending against children. Further, the items I created should be validated in the next study and hopefully used with a community or university sample. Ideally, the EASOC will provide further insight into sexual offending against children, assist correctional facilities through acting as a reliable scale in the future, and ultimately filling in the gaps for evaluative attitudes throughout the literature. 


\section{References}

Abel, G. G., Becker, J. V., \& Cunningham-Rathner, J. (1984). Complications, consent, and cognitions in sex between children and adults. International Journal of Law and Psychiatry, 7(1), 89-103. https://doi.org/10.1016/0160-2527(84)90008-6

Abel, G. G., Gore, D. K., Holland, C. L., Camp, N., Becker, J. V., \& Rathner, J. (1989). The measurement of the cognitive distortions of child molesters. Annals of Sex Research, 2(2), 135-152. https://doi.org/10.1177/107906328900200202

https://doi.org/10.1016/j.avb.2005.10.003

Beck, A. T. (1979). Cognitive therapy of depression. Guilford Press.

Bumby, K. M. (1996). Assessing the cognitive distortions of child molesters and rapists: development and validation of the MOLEST and RAPE scales. Sexual Abuse, 8(1), 3754. https://doi.org/10.1177/107906329600800105

Clark, L. A., \& Watson, D. (2019). Constructing validity: New developments in creating objective measuring instruments. Psychological Assessment, 31(12), 1412-1427. https://doi.org/10.1037/pas0000626

Coe, R. (2002, September 25). It's the effect size, stupid: What effect size is and why it is important. Education-line. https://www.leeds.ac.uk/educol/documents/00002182.htm

Government of Canada, D. of J. (2017, January 31). Sexual violations against children and child pornography. https://www.justice.gc.ca/eng/rp-pr/jr/jf-pf/2019/mar02.html

Government of Canada, S. C. (2014, May 28). Police-reported sexual offences against children and youth in Canada, 2012. https://www150.statcan.gc.ca/n1/pub/85-002x/2014001/article/14008-eng.htm 
Hermann, C. A., Nunes, K. L., \& Maimone, S. (2018). Examining implicit and explicit evaluations of sexual aggression and sexually aggressive behavior in men recruited online. Sexual Abuse, 30(5), 484-509. https://doi.org/10.1177/1079063216681560

Jahnke, S., Schmitt, S., \& Malón, A. (2018). What if the child appears to enjoy it? Moral attitudes toward Adult-child sex among men with and without pedohebephilia. The Journal of Sex Research, 55(7), 927-938. https://doi.org/10.1080/00224499.2016.1271101

Nunes, K. L., Hermann, C. A., \& Ratcliffe, K. (2013). Implicit and explicit attitudes toward rape are associated with sexual aggression. Journal of Interpersonal Violence, 28(13), 26572675. https://doi.org/10.1177/0886260513487995

Nunes, K. L. (2018, May). Evaluative attitudes toward sexual aggression. Invited workshop presented at the 17th biennial Violence and Aggression Symposium, University of Saskatchewan, Saskatoon.

Nunes, K. L., Pedneault, C. I., \& Hermann, C. A. (2021). The Evaluation of Violence Questionnaire (EVQ): Development and validity of a self-report measure of evaluative attitudes toward violence. Manuscript submitted for publication.

Nunes, K. L., Hermann, C. A., White, K., Pettersen, C., \& Bumby, K. (2018). Attitude may be everything, but is everything an attitude? Cognitive distortions may not be evaluations of rape. Sexual Abuse: A Journal of Research and Treatment, 30(1), 43-62. https://doi.org/10.1177/1079063215625489

Nunes, K. L., Hermann, C. A., Maimone, S., \& Woods, M. (2015). Thinking clearly about violent cognitions: Attitudes may be distinct from other cognitions. Journal of Interpersonal Violence, 30, 1322-1347. doi: 10.1177/0886260514540329 
Merriam-Webster. [Def. 4a, 4b]. (n.d.). Attitude. In Merriam-Webster.com dictionary. Retrieved July 17, 2020, from https://www.merriam-webster.com/dictionary/attitude

Widman, L., \& Olson, M. (2013). On the Relationship Between Automatic Attitudes and SelfReported Sexual Assault in Men. Archives of Sexual Behavior, 42(5), 813823. https://doi.org/10.1007/s10508-012-9970-2 


\section{Appendix A}

\section{ESOC Questionnaire}

Please rate how you think or feel about each of the items below. For example, how good or bad do you think it would be to do something sexual with a child?

For all the items below, "child" means someone 13 years old or younger.

Answering these questions does NOT mean that you have done or will do the things described in the questions. Please just say what you think about the idea of doing each of these things, even if you have never done them or never will do them.

You will notice that some questions are repeated with only small changes. This is not a trick or a mistake, so please answer even the similar questions.

Please circle one of the four answers under each statement.

You sexually touch a child.

very bad pretty bad not so bad not bad at all

You have an erection as you hug a child. You continue to hug the child, pressing your penis against the child.

very bad pretty bad not so bad not bad at all

A child asks you about masturbation so you touch their genitals to teach them how to masturbate.

very bad pretty bad not so bad not bad at all

A child asks about masturbation so you masturbate in front of the child to teach them how to do it.

very bad pretty bad not so bad not bad at all 
A child asks you about sex, so you sexually touch the child to teach them.

very bad pretty bad not so bad not bad at all

A child asks you what a penis is, so you show them yours and have them touch it for your sexual pleasure.

very bad pretty bad not so bad not bad at all

You are bathing a child and they ask you to wash their private parts. You wash and continue to rub their genitals to make the child feel good.

very bad pretty bad not so bad not bad at all

To prepare them for the adult world, you get two children to sexually touch each other while you watch.

very bad pretty bad not so bad not bad at all

You let a child touch your penis for their sexual pleasure.

very bad pretty bad not so bad not bad at all

A child wants to touch your penis, and you let them for your sexual pleasure.

very bad pretty bad not so bad not bad at all

You sexually touch a child who you know has been neglected or abused, to show them love. 
very bad pretty bad not so bad not bad at all

You sexually touch a child because their parents are neglecting them, and you want them to feel loved.

very bad pretty bad not so bad not bad at all

You sexually touch a shy child to help them be less shy.

very bad pretty bad not so bad not bad at all

You sexually touch a child who doesn't have a lot of friends so that they can enjoy some attention.

very bad pretty bad not so bad not bad at all

You sexually touch a child to help them feel better about their body.

very bad pretty bad not so bad not bad at all

Please circle "not so bad". (This is just to check if people are paying attention and understanding.)

Very bad pretty bad not so bad not bad at all

You see that a child is sad, so you sexually touch them to cheer them up.

very bad pretty bad not so bad not bad at all 
You tuck a child into bed at night and touch them sexually to help them get to sleep.

very bad pretty bad not so bad not bad at all

While you're drunk, you sexually touch a child.

very bad pretty bad not so bad not bad at all

Your romantic partner doesn't want to have sex with you, so you sexually touch a child instead.

very bad pretty bad not so bad not bad at all

You sexually touch a child because they won't reject you like adults will.

very bad pretty bad not so bad not bad at all

Your partner wants to sexually touch a child with you, so you do it to make your partner happy.

very bad pretty bad not so bad not bad at all

You sexually touch a child who is passed out.

very bad pretty bad not so bad not bad at all

You sexually touch a child who is sleeping deeply. 
very bad pretty bad not so bad not bad at all

You are helping a child into their bathing suit, and you touch their genitals for your sexual pleasure.

very bad pretty bad not so bad not bad at all

A child puts their hand on your penis, and you let it continue for your sexual pleasure.

very bad pretty bad not so bad not bad at all

A child sits on your lap and rubs against your penis, you let it continue for your sexual pleasure.

very bad pretty bad not so bad not bad at all

You are so horny that you sexually touch a child who is close by.

very bad pretty bad not so bad not bad at all

You are very depressed, so you sexually touch a child to try to feel better.

very bad pretty bad not so bad not bad at all

You are lonely, so you sexually touch a child to feel close to someone.

very bad pretty bad not so bad not bad at all 
You have a "tickle fight" with a child and touch their genitals for your sexual pleasure.

very bad pretty bad not so bad not bad at all

You sexually touch a child.

very negative pretty negative not so negative not negative at all

You have an erection as you hug a child. You continue to hug the child, pressing your penis against the child.

very negative pretty negative not so negative not negative at all

A child asks you about masturbation so you touch their genitals to teach them how to masturbate.

very negative pretty negative not so negative not negative at all

A child asks about masturbation so you masturbate in front of the child to teach them how to do it.

very negative pretty negative not so negative not negative at all

A child asks you about sex, so you sexually touch the child to teach them.

very negative pretty negative not so negative not negative at all

A child asks you what a penis is, so you show them yours and have them touch it for your sexual pleasure. 
very negative pretty negative not so negative not negative at all

You are bathing a child and they ask you to wash their private parts. You wash and continue to rub their genitals to make the child feel good.

very negative pretty negative not so negative not negative at all

To prepare them for the adult world, you get two children to sexually touch each other while you watch.

very negative pretty negative not so negative not negative at all

You let a child touch your penis for their sexual pleasure.

very negative pretty negative not so negative not negative at all

A child wants to touch your penis, and you let them for your sexual pleasure.

very negative pretty negative not so negative not negative at all

You sexually touch a child who you know has been neglected or abused, to show them love.

very negative pretty negative not so negative not negative at all

You sexually touch a child because their parents are neglecting them, and you want them to feel loved.

very negative pretty negative not so negative not negative at all 
You sexually touch a shy child to help them be less shy.

very negative pretty negative not so negative not negative at all

You sexually touch a child who doesn't have a lot of friends so that they can enjoy some attention.

very negative pretty negative not so negative not negative at all

You sexually touch a child to help them feel better about their body.

very negative pretty negative not so negative not negative at all

Please circle "not so negative". (This is just to check if people are paying attention and understanding.)

very negative pretty negative not so negative not negative at all

You see that a child is sad, so you sexually touch them to cheer them up.

very negative pretty negative not so negative not negative at all

You tuck a child into bed at night and touch them sexually to help them get to sleep.

very negative pretty negative not so negative not negative at all

While you're drunk, you sexually touch a child. 
very negative pretty negative not so negative not negative at all Your romantic partner doesn't want to have sex with you, so you sexually touch a child instead.

very negative pretty negative not so negative not negative at all

You sexually touch a child because they won't reject you like adults will.

very negative pretty negative not so negative not negative at all

Your partner wants to sexually touch a child with you, so you do it to make your partner happy.

very negative pretty negative not so negative not negative at all

You sexually touch a child who is passed out.

very negative pretty negative not so negative not negative at all

You sexually touch a child who is sleeping deeply.

very negative pretty negative not so negative not negative at all

You are helping a child into their bathing suit, and you touch their genitals for your sexual pleasure.

very negative pretty negative not so negative not negative at all 
A child puts their hand on your penis, and you let it continue for your sexual pleasure.

very negative pretty negative not so negative not negative at all

A child sits on your lap and rubs against your penis, you let it continue for your sexual pleasure.

very negative pretty negative not so negative not negative at all

You are so horny that you sexually touch a child who is close by.

very negative pretty negative not so negative not negative at all

You are very depressed, so you sexually touch a child to try to feel better.

very negative pretty negative not so negative not negative at all

You are lonely, so you sexually touch a child to feel close to someone.

very negative pretty negative not so negative not negative at all

You have a "tickle fight" with a child and touch their genitals for your sexual pleasure.

very negative pretty negative not so negative not negative at all 


\section{Appendix B \\ Demographic Questionnaire}

Date $(\mathrm{mm} / \mathrm{dd} /$ yyyy $)$ How old are you? years old

What is your gender? $\quad \square$ Male $\quad \square$ Female $\quad \square$ Other

What is your ethnicity? (check all that apply)

$\square$ White

$\square$ Black

$\square$ Indigenous

$\square$ Asian

$\square$ East Indian

$\square$ Hispanic

$\square$ Arab

$\square$ Other (please specify)

What kind of offenses are you in prison for now? (check all that apply)

$\square \quad$ Violent (for example, assault, robbery, making threats)

$\square$ Non-violent non-sexual (for example, theft, break and enter, drug possession)

$\square$ Sexual against a person age 18 years or older (for example, sexual assault)

$\square$ Sexual against a 16 to 17 year old person

$\square$ Sexual against a 13 to 15 year old person

$\square$ Sexual against a person age 12 years or younger

$\square$ Child pornography

Not counting the offenses that you're in prison for now, what kind of offenses were you convicted of before? (check all that apply)

$\square$ Violent (for example, assault, robbery, making threats)

․ Non-violent non-sexual (for example, theft, break and enter, drug possession)

$\square \quad$ Sexual against a person age 18 years or older (for example, sexual assault)

$\square$ Sexual against a 16 to 17 year old person

$\square$ Sexual against a 13 to 15 year old person

$\square$ Sexual against a person age 12 years or younger

$\square \quad$ Child pornography

No prior offenses 


\section{Appendix C}

\section{Consent Form for Study on Opinions About Sexual Contact with Children}

A consent form tells you what we want you to do as a participant and allows you to choose whether you want to be involved in the study or not. Consent forms also list any potential bad outcomes and they tell you who to contact in case you have any questions or concerns after the research is finished, or in case you have any questions or concerns that cannot be answered by the researcher.

Research personnel. Dr. Kevin Nunes (Professor, Department of Psychology, Carleton University, 613-520-2600, ext 1545; kevin.nunes@,carleton.ca).

Purpose. We are trying to make a new questionnaire to measure what men think about sexual contact with children, and test if that thinking is related to a history of sexual offenses against children. Common responses from you and the other participants to the questions in this study will tell us which of the questions we should keep for the final version of the questionnaire.

Task requirements. In this anonymous questionnaire, you will be asked questions about the types of offences on your record and your opinion about sexual contact with children. Participation will take about 35 minutes.

Benefits/compensation. There is no payment or other benefit to you for doing this study. Whether or not you participate will NOT affect your treatment by the institution/organization in any way.

Potential risk/discomfort. Because the questions ask about offence record and sexual contact with children, some people may feel upset. You are free to stop the survey at any time or refuse to answer any of the questions.

Anonymity/confidentiality. Your participation and answers on the questionnaire will be anonymous. In other words, nobody will be able to know who said what on the questionnaires or who completed the questionnaires.

Your answers on the questionnaire will NOT be shared with correctional staff UNLESS you write down information that we would have to report, such as plans to hurt yourself or others, or information about unknown abuse of a child. Please do not write down any information like that or any information that could identify you, like your name or a victim's name. You are safe as long as you stick to the information we ask for, so please only answer the questions we ask and do not add any other information that we do not ask you for.

The information you provide will be used only for research and teaching purposes, such as presentations at conferences and articles in scientific journals.

Because of COVID-19 restrictions, the completed questionnaires will be temporarily kept at the researcher's home before being permanently stored in a locked filing cabinet in Kevin Nunes' lab at Carleton University and on password-protected computers, and will be accessible only to the researchers working on this project and related future research.

Right to withdraw. Whether you decide to do this study or not is your choice. At any point during the study, you have the right to not complete certain questions, or to stop. If you start answering questions but then decide you want to withdraw at any point, just draw an $\mathrm{X}$ through 
your answers, leave the rest of the questionnaire blank, put the questionnaire in the envelope, seal it, and return it to Dr. Griffith.

Please note that it is not possible to withdraw your data after you have handed in the questionnaire because we will not be able to find which questionnaire is yours.

Concerns. If you have any questions or concerns about this study, please contact Kevin Nunes. If you have any ethical concerns with the study, please contact the REB Chair, Carleton University Research Ethics Board-B (by phone: 613-520-2600 ext. 4085 or by email: ethics@carleton.ca).

This study has received clearance by the Carleton University Research Ethics Board - B (CUREB-B Clearance \#112589).

If you agree to participate in the study, please answer the questions on the next pages, put them in the envelope, seal the envelope, and give it to Dr. Griffith.

If you do not want to do the study, just leave the questionnaire blank, put it in the envelope, seal the envelope, and return it to Dr. Griffith.

Appendix D

Debriefing Form

Thank you very much for participating in our study. We hope the following information addresses any questions or concerns you may have.

If you want to keep this debriefing form, please feel free to remove it before you put your questionnaire in the envelope.

PLEASE REMEMBER TO SEAL THE ENVELOPE BEFORE YOU HAND IT IN.

\section{Present study: Opinions about sexual contact with children}

\section{What are we trying to learn in this research?}

We are making a new measure of attitudes toward sexual contact with children. The purpose of the current study is to choose which of the many questions you answered should be included in the final measure. We will choose the best questions based on average responses and the correlations between the questions. We will also look at the relationship between these questions and history of sexual offences against children. We expect that attitudes will be related to a record of sexual offences against children.

\section{Why is this research important?}

Attitudes toward sexual aggression are thought to be important for sexual offending, but there is still a lot to learn (Nunes et al., 2018). This line of research will help us better understand 
attitudes toward sexual offending against children and may help people to reduce sexual offending.

Who to contact if you have questions?

The present study is being done under the supervision of Dr. Kevin Nunes (Professor, Department of Psychology, Carleton University, 613-520-2600, ext. 1545;

kevin.nunes@carleton.ca). If you have any questions or concerns about this study please contact Kevin Nunes. Should you have any ethical concerns with the study, please contact the REB Chair, Carleton University Research Ethics Board-B (by phone: 613-520-2600 ext. 4085 or by email: ethics@carleton.ca). For all other questions about the study, please contact the researcher.

Mental health resources if you found this study emotionally draining?

If you experience any distress (e.g., feel sad or mad) as a result of this study, please seek help from a staff member.

This study has been approved by the Carleton University Research Ethics Board - B (\#112589).

Thank you very much for making this research possible. 